\title{
On a generalization of the Dirac bracket in the De Donder-Weyl Hamiltonian formalism*
}

\author{
I. KANATCHIKOV* \\ Institute of Theoretical Physics, TU Berlin, \\ D-10623 Berlin, Germany \\ *E-mail: ivar@itp.physik.tu-berlin.de
}

\begin{abstract}
The elements of the contrained dynamics algorithm in the De Donder-Weyl (DW) Hamiltonian theory for degenerate Lagrangian theories are discussed. A generalization of the Dirac bracket to the DW Hamiltonian theory with second class constraints (defined in the text) is presented.

Keywords: De Donder-Weyl Hamiltonian formalism, Poisson-Gerstenhaber brackets, degenerate Lagrangians, constrained dynamics, Dirac brackets.
\end{abstract}

\section{Introduction}

In Refs. ${ }^{1-4}$ a generalization of the Poisson bracket to the De Donder-Weyl (DW) Hamiltonian formulation ${ }^{5,6}$ in field theory (and the multiparametric calculus of variations) has been proposed. It leads to a Gerstenhaber algerba of brackets defined on specific horizontal differential forms (called Hamiltonian forms). The construction assumes that the corresponding Lagrangian theory is regular (in the sense of DW Hamiltonian formulation):

$$
\operatorname{det}\left\|\frac{\partial^{2} L}{\partial \phi_{a}^{\mu} \partial \phi_{a}^{\nu}}\right\| \neq 0
$$

However, many field theories of physical interests, such as spinor fields, are not regular in the above sense. In this paper we discuss a corresponding extension of the formalism to the degenerate case and generalize the above mentioned Poisson-Gerstenhaber bracket similarly to the construction of the Dirac bracket in the constrained systems with second-class constraints. ${ }^{7}$

Note that the regularity condition in the DW Hamiltonian formalism is different from the one in the standard Hamiltonian formalism:

*Proc. 10th Int. Conf. on Differential Geometry and its Applications DGA 2007, Olomouc, Czech Republic. 
$\operatorname{det}\left\|\partial^{2} L / \partial \dot{\phi}_{a} \partial \dot{\phi}_{a}\right\| \neq 0$, which involves only the time derivatives of fields $\dot{\phi}_{a}$. As a consequence, the theories which are regular from the point of view of the DW formalism can be irregular from the point of view of the standard Hamiltonian formalism and vice versa.

This work is motivated by the project of manifestly space-time symmetric precanonical quantization of field theor ${ }^{8-11}$ based on the DW Hamiltonian formalism, which requires a well understood formalism for degenerate theories on the classical level.

\section{The polysymplectic structure and the Poisson-Gerstenhaber brackets}

In this section we briefly recall the construction and properties of the Poisson-Gerstenhaber brackets in DW Hamiltonian formalism which are used in the following section. For further generalizations and a detailed geometrical treatment of the related issues I refer to the existing literature. ${ }^{12-17}$ Here I closely follow my previous work. ${ }^{2-4,10}$

The DW Hamiltonian formalism ${ }^{5,6}$ is a space-time symmetric generalization of the Hamiltonian formalism from mechanics to field theory. Given the first order Lagrangian density $L=L\left(y^{a}, y_{\mu}^{a}, x^{\nu}\right)$ we introduce the variables (polymomenta)

$$
p_{a}^{\mu}:=\partial L / \partial y_{\mu}^{a}
$$

and the DW Hamiltonian function

$$
H:=y_{\mu}^{a} p_{a}^{\mu}-L=H\left(y^{a}, p_{a}^{\mu}, x^{\mu}\right) .
$$

Then the Euler-Lagrange equations take the form

$$
\partial_{\mu} y^{a}(x)=\partial H / \partial p_{a}^{\mu}, \quad \partial_{\mu} p_{a}^{\mu}(x)=-\partial H / \partial y^{a},
$$

which is equivalent to the Euler-Lagrange field equations if $L$ is regular in the sense of Eq. (1).

Geometrically, classical fields $y^{a}=y^{a}(x)$ are sections in the covariant configuration bundle $Y \rightarrow X$ over an oriented $n$-dimensional spacetime manifold $X$ with the volume form $\omega$. The local coordinates in $Y$ are $\left(y^{a}, x^{\mu}\right)$. If $\bigwedge_{q}^{p}(Y)$ denotes the space of $p$-forms on $Y$ which are annihilated by $(q+1)$ arbitrary vertical vectors of $Y$, then $\bigwedge_{1}^{n}(Y) \rightarrow Y$ generalizes the cotangent bundle and serves as a model of a multisymplectic phase space 6 which possesses the canonical $n$-form structure

$$
\Theta_{M S}=p_{a}^{\mu} d y^{a} \wedge \omega_{\mu}+p \omega
$$


called multisymplectic, where $\omega_{\mu}:=\partial_{\mu} \downarrow \omega$ are the basis of $\bigwedge^{n-1} T^{*} X$. A section $p=-H\left(y^{a}, p_{a}^{\mu}, x^{\nu}\right)$ yields the multidimensional Hamiltonian Poincaré-Cartan form $\Theta_{P C}$.

In order to introduce the Poisson brackets which reflect the dynamical structure of DW Hamiltonian equations (2) we need a structure which is independent of $p$ or a choice of $H$. We define the extended polymomentum phase space as the quotient bundle $Z: \bigwedge_{1}^{n}(Y) / \bigwedge_{0}^{n}(Y) \rightarrow Y$. The local coordinates on $Z$ are $\left(y^{a}, p_{a}^{\nu}, x^{\nu}\right)=:\left(z^{v}, x^{\mu}\right)=z^{M}$. We introduce a canonical structure on $Z$ as an equivalence class of forms $\Theta:=$ $\left[p_{a}^{\mu} d y^{a} \wedge \omega_{\mu} \quad \bmod \bigwedge_{0}^{n}(Y)\right]$. The polysymplectic structure on $Z$ is defined as an equivalence class of forms $\Omega$ given by

$$
\Omega:=\left[d \Theta \bmod \bigwedge_{1}^{n+1}(Y)\right]=\left[-d y^{a} \wedge d p_{a}^{\mu} \wedge \omega_{\mu} \bmod \bigwedge_{1}^{n+1}(Y)\right] .
$$

The equivalence classes are introduced as an alternative to the explicit introduction of a non-canonical connection on the multisymplectic phase space in order to define the polysymplectic structure as a "vertical part" of the multisymplectic form $d \Theta_{M S}$. The fundamental constructions, such as the Poisson bracket below, are designed to be independent of the choice of representatives in the equivalence classes and the choice of a connection.

A multivector field of degree $p, \stackrel{p}{X} \in \bigwedge^{p} T Z$, is called vertical if $\left.\stackrel{p}{X}\right\lrcorner F=$ 0 for any form $F \in \bigwedge_{0}^{*}(Z)$. The polysymplectic form establishes a map of horizontal $p$-forms $\stackrel{p}{F} \in \bigwedge_{0}^{p}(Z), p=0,1, \ldots,(n-1)$, to vertical multivector fields of degree $(n-p), \stackrel{n-p}{X}_{F}$, called Hamiltonian:

$$
\left.\stackrel{n-p}{X}_{F}\right\lrcorner \Omega=d \stackrel{p}{F} .
$$

The forms for which the map (5) exists are also called Hamiltonian. More precisely, horizontal forms are mapped to the equivalence classes of Hamiltonian multivector fields modulo the characteristic multivector fields $\stackrel{p}{X_{0}}$ : $\left.\stackrel{p}{X_{0}}\right\lrcorner \Omega=0, p=2, \ldots, n$. It is important to note that the space of Hamiltonian forms is not stable with respect to the exterior product. The natural product operation of Hamiltonian forms is the co-exterior product

$$
\stackrel{p}{F} \bullet \stackrel{q}{F}:=*^{-1}(* \stackrel{p}{F} \wedge * \stackrel{q}{F})
$$

which is graded commutative and associative.

The Poisson bracket of Hamiltonian forms is given by the formula

$$
\left.\left.\left.\left\{\stackrel{p}{F_{1}}, \stackrel{q}{F}_{2}\right\}=(-1)^{(n-p)} \stackrel{n-p}{X}_{1}\right\rfloor d \stackrel{q}{F}_{2}=(-1)^{(n-p)} \stackrel{n-p}{X}_{1}\right\lrcorner \stackrel{n-q}{X}_{2}\right\rfloor \Omega .
$$


In fact, it is induced by the Schouten-Nijenhuis bracket $\llbracket, \rrbracket$ of the corresponding Hamiltonian multivector fields:

$$
-d\{\stackrel{p}{F}, \stackrel{q}{F}\}:=\llbracket \stackrel{n-p}{X}, \stackrel{n-q}{X} \rrbracket\lrcorner \Omega .
$$

The algebraic properties of the bracket are summarized in the following

Theorem 2.1. The space of Hamiltonian forms with the operations $\{$, $\}$ and $\bullet$ is a (Poisson-)Gerstenhaber algebra, i.e.

$$
\begin{aligned}
& \left.\{\stackrel{p}{F}, \stackrel{q}{F}]\}=-(-1)^{g_{1} g_{2}}\{\stackrel{q}{F}, \stackrel{p}{F}]\right\}, \\
& \left.\left.(-1)^{g_{1} g_{3}}\{\stackrel{p}{F},\{\stackrel{q}{F}, \stackrel{r}{F}\}\}\right\}+(-1)^{g_{1} g_{2}}\{\stackrel{q}{F},\{\stackrel{r}{F}, \stackrel{p}{F}]\}\right\} \\
& \left.+(-1)^{g_{2} g_{3}}\{\stackrel{r}{F},\{\stackrel{p}{F}, \stackrel{q}{F}\}\}\right\}=0, \\
& \left.\{\stackrel{p}{F}, \stackrel{q}{F} \bullet \stackrel{r}{F}\}\}=\{\stackrel{p}{F}, \stackrel{q}{F}\}\} \bullet \stackrel{r}{F}+(-1)^{g_{1}\left(g_{2}+1\right)} \stackrel{q}{F} \bullet\{\stackrel{p}{F}, \stackrel{r}{F}\}\right\},
\end{aligned}
$$

where $g_{1}=n-p-1, g_{2}=n-q-1, g_{3}=n-r-1$.

The graded Lie algebra properties are induces by the graded Lie properties of the Schouten-Nijenhuis bracket. The graded Leibniz property is a consequence of the Frölicher-Nijenhuis classification of graded derivations adapted to the co-exterior algebra of forms.

Let us mention a few applications of the Poisson-Gerstenhaber (PG) brackets. They enable us to identify the pairs of canonically conjugate variables which may become a starting point of quantization:

$$
\left\{p_{a}^{\mu} \omega_{\mu}, y^{b}\right\}=\delta_{a}^{b}, \quad\left\{p_{a}^{\mu} \omega_{\mu}, y^{b} \omega_{\nu}\right\}=\delta_{a}^{b} \omega_{\nu}, \quad\left\{p_{a}^{\mu}, y^{b} \omega_{\nu}\right\}=\delta_{a}^{b} \delta_{\nu}^{\mu} .
$$

In fact, geometric prequantization of PG brackets has been discussed in our previous paper. ${ }^{10}$ The brackets can be used also in order to write the DW Hamiltonian equations in the bracket form: for any Hamiltonian ( $n-$ 1)-form $F:=F^{\mu} \omega_{\mu}$ the equations of motion take the form

$$
\mathbf{d} \bullet F=-\sigma(-1)^{n}\{H, F\}+d^{h} \bullet F,
$$

where $\mathbf{d} \bullet$ denotes the "total co-exterior differential"

$$
\left.\mathbf{d} \bullet \stackrel{p}{F}:=\frac{1}{(n-p) !} \partial_{M} F^{\mu_{1} \ldots \mu_{n-p}} \partial_{\mu} z^{M} d x^{\mu} \bullet \partial_{\mu_{1} \ldots \mu_{n-p}}\right\lrcorner \omega,
$$

$d^{h}$ is the "horizontal co-exterior differential":

$$
\left.d^{h} \bullet \stackrel{p}{F}:=\frac{1}{(n-p) !} \partial_{\mu} F^{\mu_{1} \ldots \mu_{n-p}} d x^{\mu} \bullet \partial_{\mu_{1} \ldots \mu_{n-p}}\right\rfloor \omega,
$$

and $\sigma= \pm 1$ for the Euclidean/Minkowskian signature of the base manifold $X$. Consequently, the conservation of the quantity represented by $(n-1)$ form $F$ is equivalent to the condition $\{H, F\}=0$. 


\section{The constrained dynamics and the Dirac bracket of forms}

The violation of the regularity condition (1) implies the noninvertibility of the space-time gradients of fields $\phi_{\nu}^{a}$ as functions of the field variables $\phi^{a}$ and polymomenta $p_{a}^{\nu}$. In this case the polymomenta are not all independent, and there exist relations

$$
C_{A}\left(\phi^{a}, p_{a}^{\nu}\right)=0
$$

that follow from the definition of the polymomenta and the form of the Lagrangian. The conditions (15), as usual, will be called primary constraints to emphasize that the field equations are not used to obtain these relations and that they imply no restriction on the field variables and their space-time gradients.

The canonical DW Hamiltonian function is now not unique. It can be replaced by the effective DW Hamiltonian function which is weakly equal to $H$ :

$$
\tilde{H}:=H+u_{A} C_{A} \approx H .
$$

In the present approach we restrict our attention to the constraints which can be organized into Hamiltonian $(n-1)$-forms

$$
\mathfrak{C}_{m}=C_{m}^{\mu} \omega_{\mu}
$$

In this case the effective Hamiltonian can be written as

$$
\tilde{H}:=H+u_{m} \bullet \mathfrak{C}_{m},
$$

where the Lagrange multipliers are organized into one-forms $u_{m}$.

Definition 3.1. A dynamical variable represented by a semi-basic Hamiltonian form $F$ of degree $|F|=f$ is said to be first class if its $P G$ bracket with every constraints $(n-1)$-form $\mathfrak{C}_{m}$ weakly vanishes,

$$
\left\{F, \mathfrak{C}_{m}\right\} \approx 0 .
$$

Definition 3.2. A semi-basic Hamiltonian form $F$ is said to be second class if there is at least one constraint such that its $P G$ bracket with $F$ does not vanish weakly.

Proposition 3.1. The first-class property is preserved under the $P G$ bracket operation, i.e. the $P G$ bracket of two first-class Hamiltonian forms is first class. 
Proof. If $F$ and $G$ are first class, then

$$
\left.\left.\left\{F, \mathfrak{C}_{m}\right]\right\}=f_{m}^{n} \bullet \mathfrak{C}_{n}, \quad\left\{G, \mathfrak{C}_{m}\right]\right\}=g_{m}^{n} \bullet \mathfrak{C}_{n}
$$

for some $(f+1)$-forms $f_{m}^{n}$ and $(g+1)$-form $g_{m}^{n}$, where $|G|=g,|F|=f$. By the graded Jacobi identity

$-(-1)^{d_{G}}\left\{\left\{\mathfrak{C}_{m},\{F, G]\right\}=(-1)^{d_{F}}\left\{F,\left\{\left[G, \mathfrak{C}_{m}\right\}\right\}\right\}+(-1)^{d_{F} d_{G}}\left\{\left[G,\left\{\left[\mathfrak{C}_{m}, F\right]\right\}\right\}\right.\right.$,

where $d_{G}:=n-g-1, d_{F}:=n-f-1$. Using Eq. (20), the graded Leibniz rule, and the graded antisymmetry of the bracket in the right hand side, we obtain

$$
\left.(-1)^{d_{G}}\left\{F, g_{m}^{n} \bullet \mathfrak{C}_{n}\right]\right\}-(-1)^{d_{F} d_{G}+d_{F}}\left\{\left[G, f_{m}^{n} \bullet \mathfrak{C}_{n}\right]\right\} \approx 0 .
$$

Thus,

$$
\left.\left\{\mathfrak{C}_{m},\{F, G]\right\}\right\} \approx 0
$$

for any first class Hamiltonian forms $F$ and $G$.

Next, we consider the necessary condition for the conservation of the constraints $\mathfrak{C}_{m}$ forms : $\mathbf{d} \bullet \mathfrak{C}_{m}=0$ (c.f. Eq. (12)). They give rise to the necessary consistency conditions

$$
\left\{\tilde{H}, \mathfrak{C}_{m}\right\}=\left\{\left[H, \mathfrak{C}_{m}\right\}+u^{n} \bullet\left\{\mathfrak{C}_{n}, \mathfrak{C}_{m}\right\}\right\} 0,
$$

where $u^{n}$ are one-form coefficients. Eq. (21) can either impose a restriction on the $u$ 's or it may reduce to a new relation independent of the $u$ 's. In the latter case, i.e. if the new relation between $p$ 's and $\phi$ 's is independent of the primary constraints, it will be called a secondary constraint, in accordance with the conventional terminology introduced by Dirac and Bergmann.

While the primary constraints are merely consequences of the definition of the polymomentum variables, the secondary constraints make use of the equations of motion (the DW Hamiltonian field equations) as well. If there is a secondary constraint written as an $(n-1)$-form $\mathfrak{B}_{m}(\phi, p):=B_{m}^{\mu} \omega_{\mu}$ appearing, a new consistency condition in the form (21)

$$
\left.\left\{H, \mathfrak{B}_{m}\right\}+u^{n} \bullet\left\{\mathfrak{B}_{n}, \mathfrak{C}_{m}\right\}\right\} 0
$$

must be imposed. Next, we must again check whether Eq. (22) implies new secondary constraints or whether it only restructures the $u$ 's, and so on. It is similar to the standard procedure originally proposed by Dirac. 
The second-class constraints are present whenever the $(n-1)$-form valued matrix

$$
\mathfrak{C}_{m n}:=\left\{\mathfrak{C}_{m}, \mathfrak{C}_{n}\right\}
$$

does not vanish on the constraints surface. As usual, when computing $\mathfrak{C}_{m n}$ we must not use the constraints equations until after calculating the Poisson-Gerstenhaber bracket. For simplicity, we assume that the constraints are irreducible and the rank of $\mathfrak{C}_{m n}$ is constant on the constraints surface. Since $\mathfrak{C}_{m n}$ is a nonsingular matrix whose components are $(n-1)$ forms, its "inverse" $\mathfrak{C}_{m n}^{-1}$ exists such that

$$
\mathfrak{C}_{m n}^{-1} \wedge \mathfrak{C}_{n k}=\delta_{m k} \omega
$$

The components of $\mathfrak{C}_{m n}^{-1}$ are one-forms. The key observation is that

Proposition 3.2. For any Hamiltonian form $F$ we can construct a new Hamiltonian form $F^{\prime} \approx F$,

$$
F^{\prime}:=F-\sigma\left\{F, \mathfrak{C}_{m}\right\} \bullet\left(\mathfrak{C}_{m n}^{-1} \wedge \mathfrak{C}_{n}\right),
$$

which has vanishing brackets with all second class constraints.

Here, $\sigma$ is the signature of the metric whose appearence will be clarified later. The parentheses in Eq. (25) are important because the algebraic system of exterior forms equipped with two products $\wedge$ and $\bullet$ is not associative.

Proof. Using the (graded) Leibniz rule with respect to the •-product, which is fulfilled by the Poisson-Gerstenhaber bracket of Hamiltonian forms, we obtain

$$
\begin{aligned}
\left.\left\{F^{\prime}, \mathfrak{C}_{k}\right]\right\} & =\left\{\left[F, \mathfrak{C}_{k}\right\}-\sigma\left\{\left\{f F, \mathfrak{C}_{m}\right\} \bullet\left(\mathfrak{C}_{m n}^{-1} \wedge \mathfrak{C}_{n}\right), \mathfrak{C}_{k}\right]\right\} \\
& =\left\{\left[F, \mathfrak{C}_{k}\right\}-\sigma\left\{F, \mathfrak{C}_{m}\right\} \bullet\left\{\mathfrak{C}_{m n}^{-1} \wedge \mathfrak{C}_{n}, \mathfrak{C}_{k}\right\}\right. \\
& -\sigma\left\{\left\{\left[F, \mathfrak{C}_{m}\right]\right\}, \mathfrak{C}_{k}\right\} \bullet\left(\mathfrak{C}_{m n}^{-1} \wedge \mathfrak{C}_{n}\right) .
\end{aligned}
$$

As the last term weakly vanishes,

$$
\left.\left\{F^{\prime}, \mathfrak{C}_{k}\right\}\right]\left\{\left\{F, \mathfrak{C}_{k}\right\}\right\}-\sigma\left\{F, \mathfrak{C}_{m}\right\} \bullet\left\{\mathfrak{C}_{m n}^{-1} \wedge \mathfrak{C}_{n}, \mathfrak{C}_{k}\right\} .
$$

Let us consider the bracket

$$
\left\{\mathfrak{C}_{k}, \mathfrak{C}_{m n}^{-1} \wedge \mathfrak{C}_{n}\right\}=-£_{X_{\mathfrak{c}_{k}}}\left(\mathfrak{C}_{m n}^{-1} \wedge \mathfrak{C}_{n}\right),
$$


where $£_{X_{\mathfrak{C}_{k}}}$ is the Lie derivative with respect to the vector field $X_{\mathfrak{C}_{k}}$ associated with the Hamiltonian form $\mathfrak{C}_{k}$ via the correspondence given by the polysymplectic form $\Omega$ :

$$
\left.X_{\mathfrak{C}_{k}}\right\lrcorner \Omega=d \mathfrak{C}_{k}
$$

(c.f. Eq. (7)). As the Lie derivative is a derivation on the $\wedge$-algebra,

$$
\left\{\mathfrak{C}_{k}, \mathfrak{C}_{m n}^{-1} \wedge \mathfrak{C}_{n}\right\}=-£_{X_{\mathfrak{C}_{k}}}\left(\mathfrak{C}_{m n}^{-1}\right) \wedge \mathfrak{C}_{n}-\mathfrak{C}_{m n}^{-1} \wedge £_{X_{\mathfrak{c}_{k}}}\left(\mathfrak{C}_{n}\right)
$$

we obtain

$$
\left\{\{ \mathfrak { C } _ { k } , \mathfrak { C } _ { m n } ^ { - 1 } \wedge \mathfrak { C } _ { n } \} \approx \mathfrak { C } _ { m n } ^ { - 1 } \wedge \left\{\left\{\mathfrak{C}_{k}, \mathfrak{C}_{n}\right\}=-\mathfrak{C}_{m n}^{-1} \wedge \mathfrak{C}_{n k}=-\delta_{m k} \omega .\right.\right.
$$

Further, using $* \omega=\sigma$, so that for any form $F: F \bullet \omega=\sigma F$, we obtain

$$
\left.\left\{F^{\prime}, \mathfrak{C}_{k}\right]\right\} \approx\left\{F, \mathfrak{C}_{k}\right\}-\sigma\left\{F, \mathfrak{C}_{m}\right] \bullet \bullet \delta_{m k} \omega=\left\{F, \mathfrak{C}_{k}\right\}-\sigma^{2}\left\{F, \mathfrak{C}_{k}\right\}=0 .
$$

Hence,

$$
\left.\left\{F^{\prime}, \mathfrak{C}_{k}\right]\right\} \approx 0
$$

Now, following a well known way of introducing the Dirac bracket, we may try to define it as $\left\{F^{\prime}, G^{\prime}\right\}$. Though $F^{\prime} \approx F$ and $G^{\prime} \approx G$, the bracket $\left\{F^{\prime}, G^{\prime}\right\}$ is not weakly equal to $\{F, G\}$ and the desired properties

$$
\left.\left\{F^{\prime}, G^{\prime}\right\} \approx\left\{F^{\prime}, G\right]\right\} \approx\left\{F, G^{\prime}\right\}
$$

are satisfied if $F$ and $G$ have the degree $(n-1)$. In this case we obtain the following formula for the analogue of the Dirac bracket

$$
\left.\{F, G\}^{D}:=\{F, G\}\right\}-\sigma\left\{F, \mathfrak{C}_{m}\right\} \bullet\left(\mathfrak{C}_{m n}^{-1} \wedge\left\{\left\{\mathfrak{C}_{n}, G\right\}\right) .\right.
$$

The properties of the bracket (33) are described by the following

Proposition 3.3. The Dirac bracket of any Hamiltonian (n-1)- form with a second class constraint vanishes.

Proof. The statement follows from Eq. (32) and Proposition 3.2.

Proposition 3.4. The Dirac bracket fulfills the Lie algebra properties

$$
\begin{gathered}
\left.\{F, G\}^{D} \approx-\{G, F]\right\}^{D}, \\
\left\{\left\{\{F, G\}^{D}, K\right\}^{D}+\left\{\left\{\{G, K\}^{D}, F\right\}^{D}+\left\{\{\{K, F\}\}^{D}, G\right\}^{D} \approx 0 .\right.\right.
\end{gathered}
$$


Proof. These weak identities follow from the properties of the PoissonGerstenhaber bracket in Theorem 2.1 and Eq. (32).

The space of Hamiltonian $(n-1)$ forms on which the bracket has been defined is not closed with respect the product operations $\bullet$ and $\wedge$. We, therefore, can not discuss the Leibniz property without extending the space of forms. A minimal extension would involve forms of degree 0 or $n$. Let us extend the definition of the bracket in Eq. (33) to the case when one of the arguments is a 0 -form $k$ (the bracket of two 0 -forms vanishes identically):

$$
\{F, k\}\}^{D}=\{F, k]-\sigma\left\{F, \mathfrak{C}_{m}\right\} \bullet\left(\mathfrak{C}_{m n}^{-1} \wedge\left\{\mathfrak{C}_{n}, k\right\}\right) .
$$

Then the following analogues of the Leibniz property follow:

$$
\begin{aligned}
\{[F, k G\}\}^{D} & =\{F, k\}\}^{D} G+k\{F, G\}^{D}, \\
\{[F, k l\}\}^{D} & \left.=\{[F, k\}\}^{D} l+k\{F, l\}\right\}^{D} .
\end{aligned}
$$

The Leibniz properties for the $\bullet-$ multiplication with $n$-forms are similar because $F \bullet k \omega=\sigma k F$.

\section{Conclusion}

We presented a generalization of the Dirac constrained dynamics algorithm and a generalization of the Dirac bracket formula to the De Donder-Weyl Hamiltonian formalism of degenerate Lagranigian theories. The Dirac bracket is defined for dynamical variables given by Hamiltonian $(n-1)$-forms if the constraints can also be organized into Hamiltonian $(n-1)$-forms. A possible generalization to forms of arbitrary degree and non-Hamiltonian forms remains an open issue. We also left beyond the scope of our discussion a possible geometrical interpetation of the construction in terms of the restriction of the polysymplectic structure to the subspace of constraints.

Let us recall that in the geometric calculus of variations the issues related to the degeneracy of the Lagrangian can be treated with the help of Lepagean equivalents of the Cartan form ${ }^{19}$ which effectively modify the definitions of polymomentum variables and the corresponding regularity conditions. It would be interesting to understand if our formal Dirac-like treatment of constraints in the DW theory could be understood geometrically using the theory of Lepagean equivalents. Yet another promising approach to the geometrical undertanding of the formalism could be based on the extension of the Cartan form to the constrained variational problems as presented in Ref. ${ }^{20}$ 
Note in conclusion, that the approach developed here can be applied to the Dirac spinor field ${ }^{21}$ which is a singular theory with second-class constraints from the point of view of the DW Hamiltonian formulation.

\section{Acknowledgments}

I thank Prof. Hellwig for his warm hospitality at TU Berlin. A discussion with Prof. Krupková is gratefully acknowledged.

\section{References}

1. I.V. Kanatchikov, On the canonical ctructure of the De Donder-Weyl covariant Hamiltonian formulation of field theory I. Graded Poisson brackets and the equations of motion, hep-th/9312162.

2. I.V. Kanatchikov, Canonical structure of classical field theory in the polymomentum phase space, Rep. Math. Phys. 41 (1998) 49-90, hep-th/9709229.

3. I.V. Kanatchikov, On field theoretic generalizations of a Poisson algebra, Rep. Math. Phys. 40 (1997) 225-34, hep-th/9710069.

4. I.V. Kanatchikov, Novel algebraic structures from the polysymplectic form in field theory, hep-th/9612255.

5. Th. De Donder, Théorie Invariantive du Calcul des Variations, GauthierVillars, Paris (1935);

H. Weyl, Geodesic fields in the calculus of variations, Ann. Math. (2) 36, 607-29 (1935);

H. Rund, The Hamilton-Jacobi Theory in the Calculus of Variations, D. van Nostrand, Toronto (1966).

6. M.J. Gotay, J. Isenberg and J. Marsden, Momentum maps and classical relativistic fields, Part I: Covariant field theory, physics/9801019, Part II: Canonical Analysis of Field Theories, math-ph/0411032.

7. M. Henneaux and C. Teitelboim, Quantization of Gauge Systems, Princeton Univ. Press, Princeton, NJ 1992.

8. I.V. Kanatchikov, De Donder-Weyl theory and a hypercomplex extension of quantum mechanics to field theory, Rep. Math. Phys. 43 (1999) 157-70, hep-th/9810165;

I.V. Kanatchikov, On quantization of field theories in polymomentum variables, hep-th/9811016.

9. I.V. Kanatchikov, Precanonical quantum gravity: quantization without the space-time decomposition, Int. J. Theor. Phys. 40 (2001) 1121-49, gr-qc/0012074.

10. I.V. Kanatchikov, Geometric (pre)quantization in the polysymplectic approach to field theory, hep-th/0112263.

11. I.V. Kanatchikov, Precanonical quantization of Yang-Mills fields and the functional Schrödinger representation, Rep. Math. Phys. 53 (2004) 181-193, hep-th/0301001.

12. N. Roman-Roy, A. M. Rey, M. Salgado and S. Vilarino, On the k-Symplectic, 
k-Cosymplectic and Multisymplectic Formalisms of Classical Field Theories, arXiv:0705.4364 [math-ph].

13. M. Francaviglia, M. Palese and E. Winterroth, A new geometric proposal for the Hamiltonian description of classical field theories, math-ph/0311018.

14. M. Forger, C. Paufler, H. Römer, Hamiltonian multivector fields and poisson forms in multisymplectic field theory, J. Math. Phys. 46 (2005) 112901, math-ph/0407057.

15. M. de León, M. McLean, L. K. Norris, A. Rey-Roca, M. Salgado, Geometric structures in field theory, math-ph/0208036;

A. Echeverria-Enriquez, M. de León, M. C. Munoz-Lecanda and N. Roman-Roy, Hamiltonian systems in multisymplectic field theories, arXiv:math-ph/0506003.

16. O. Krupková, Hamiltonian field theory, J. Geom. Phys. 43 (2002) 93-132.

17. G. Giachetta, L. Mangiarotti and G. Sardanashvily, New Lagrangian and Hamiltonian Methods in Field Theory, World Scientific, Singapore 1997.

18. F. Hélein, J. Kouneiher, Covariant Hamiltonian formalism for the calculus of variations with several variables, math-ph/0211046.

19. O. Krupková and D. Smetanová, Legendre transformation for regularizable Lagrangians in field theory, Lett. Math. Phys. 58 (2001) 189-204;

O. Krupková and D. Smetanová, On regularization of variational problems in first-order field theory, Rend. Circ. Mat. Palermo (2) Suppl. No. 66 (2001), 133-140;

D. Smetanová, Hamiltonian systems in dimension 4, to appear in Proc. 10th Int. Conf. Diff. Geom. and Its Applications, Olomouc, Czech Republic (2007).

20. A. Garcia, P.L. García and C. Rodrigo, Cartan forms for first order constrained variational problems, J. Geom. Phys. 56 (2006) 571-610.

21. I. Kanatchikov, in preparation. 